\title{
Aorto-Pulmonary Artery Fistula Following Aortic Valve Replacement: A Diagnostic Dilemma
}

\author{
Tarun Raina Ramman*, Shyamveer Khangarot, Sunil Kumar, M. A. Geelani, D. K. Satsangi \\ Department of Cardiothoracic and Vascular Surgery, GIPMER Hospital, New Delhi, India \\ Email: "tarunraina.25@gmail.com
}

Received 24 October 2015; accepted 25 March 2016; published 29 March 2016

Copyright $(02016$ by authors and Scientific Research Publishing Inc.

This work is licensed under the Creative Commons Attribution International License (CC BY).

http://creativecommons.org/licenses/by/4.0/

(c) (i) Open Access

\begin{abstract}
Aorto-pulmonary artery fistula is a rare and lethal occurrence after thoracic surgery and aortic aneurysms. Here we present a rare occurrence of acute aorto-pulmonary artery fistula in outpatient department in a follow up patient of aortic valve replacement and discuss challenges faced in diagnosis, etiopathogenesis and management.
\end{abstract}

\section{Keywords}

Aorto-Pulmonary Fistula, Aortic Valve Replacement, Rare, Complications

\section{Introduction}

An acquired aorto-pulmonary artery fistula is essentially a rare condition with very low chances of survival. It presents with an acute onset with rapid deterioration. Here we present the case of a 42-year-old male patient who is a follow up case of aortic valve replacement presenting with sudden onset aorto-pulmonary fistula on his follow up visit after a month.

\section{Case Report}

A 42-year male was diagnosed with bicuspid aortic valve with severe AS at GIPMER hospital New Delhi. The gradient across the aortic valve was $100 \mathrm{~mm} \mathrm{Hg}$ and the coronary angiography was within normal limits. Patients underwent aortic valve replacement size 18 ATS. The post operative course was uneventful and patient was discharged on day 10 post op. The first follow up was conducted on the 14th day post operatively and was

${ }^{*}$ Corresponding author.

How to cite this paper: Ramman, T.R., Khangarot, S., Kumar, S., Geelani, M.A. and Satsangi, D.K. (2016) Aorto-Pulmonary Artery Fistula Following Aortic Valve Replacement: A Diagnostic Dilemma. World Journal of Cardiovascular Surgery, 6, 40-43. http://dx.doi.org/10.4236/wjcs.2016.63007 
found to be uneventful. The patient was called for the 2 nd follow 1 month post operatively. While waiting for his turn in the outpatient department he developed sudden onset dypnea and tachycardia. There was no history of fever. Sudden onset dyspnea was NYHA grade 4. A systolic blood pressure of $84 \mathrm{~mm} / \mathrm{HG}$ and diastolic of 60 $\mathrm{mm} / \mathrm{Hg}$ was recorded. The heart was recorded as 144 b.p.m. On auscultation a systolic murmur was heard in aortic area and there was a loss of valve click. In view of hemodynamic instability and examination findings patient was immediately shifted to ICU with a provisional diagnosis of stuck aortic valve or aortic dissection. The transthoracic echocardiography findings were suggestive of increased gradient across aortic valve and decreased movement of the valve leaflets. There was no evidence dissection flap. The arterial blood gas revealed metabolic acidosis due to decreased peripheral perfusion. Fluoroscopy done showed normal disc movements. Hence the diagnosis was changed to acute coronary event with left ventricular failure and pulmonary edema. Patient was treated with diuretics and ionotropes. The acute coronary event was not substantiated by enzymes and patient condition kept on deteriorating. Endo tracheal intubation was done on day one of admission and patient put on ventilator support. To ascertain the diagnosis TEE was done which showed suspicion of stuck leaflet with turbulence in aorta and pulmonary artery. Because of poor hemodynamics at that moment the gradient could not be assessed.

In view of poor hemodynamic and suspicion of stuck leaflet patient was taken for emergency redo aortic valve replacement. Patient was put on femoro-femoral bypass electively and chest was opened. After adhesiolysis aorta and pulmonary artery were exposed. PA was tense with palpable thrill over PA. There was no evidence of any aneurysm. After dissecting plane between aorta and pulmonary artery, aorta was cross clamped and aortotomy was done. Cardioplegia was given through coronary ostia.

Both valve leaflets were freely mobile and there was no evidence of vegetation, thrombus or pannus. To our surprise a communication between the aorta and pulmonary artery was seen just $2 \mathrm{~cm}$ above the commissure of RCC and LCC (Figure 1). The margins of the communication were rugged and irregular with friable tissues. Pulmonary artery was opened and communication probed. There was no evidence of vegetation in pulmonary artery as well. The defect was freshened and closed with 4-o polypropylene interrupted sutures using PTFE patch from the pulmonary side. The pulmonary artery and aorta were closed, de airing was done and patient was gradually weaned off by pass. The patient was shifted to ICU with borderline hemodynamic state with ionotropic support. Unfortunately the patient deteriorated and died on post operative day 1 in spite of both inotropic and IABP support. The immediate underlying cause of death was low cardiac output syndrome.

The relatives did not consent for the autopsy of the patient. The culture report of the margin of the fistula showed no bacterial or fungal growth.

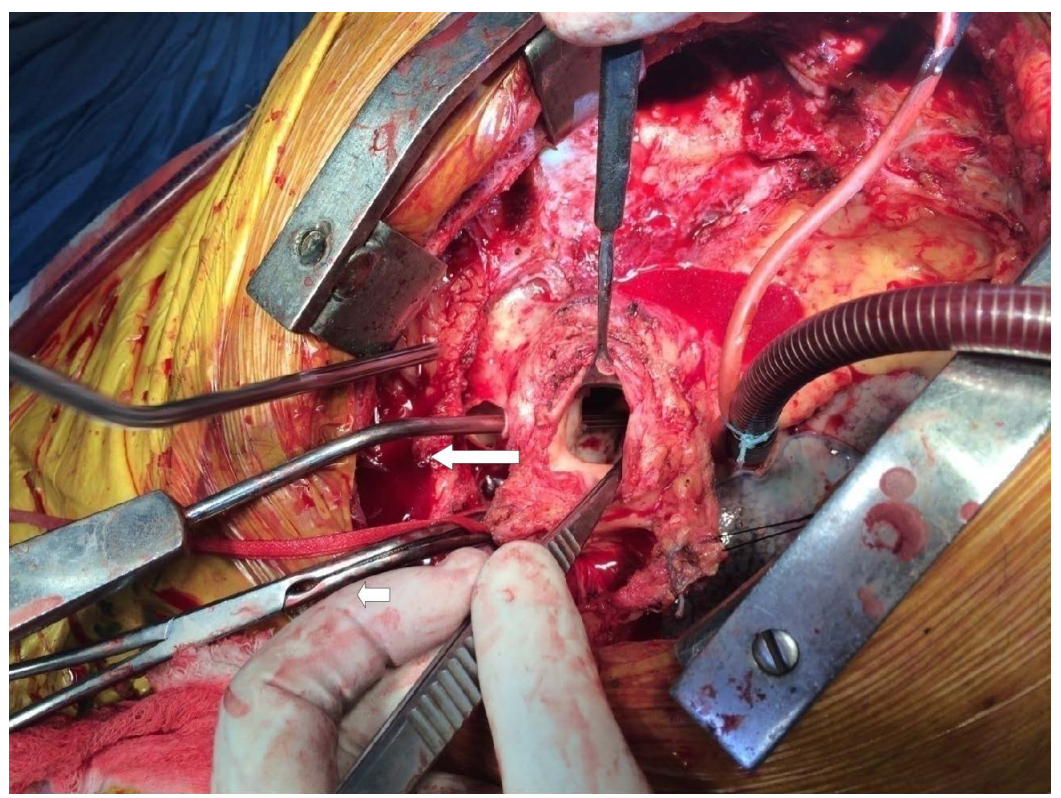

Figure 1. Showing pump sucker (long horizontal arrow) passing through the fistula from pulmonary artery end into aortic end. Small horizontal arrow marking aortic cross clamp. 


\section{Discussion}

The most common postulated causes of an aorto-pulmonary artery fistula are iatrogenic (thoracic aortic surgery), ruptured aortic arch aneurysms (often caused due to atherosclerosis and connective tissue disorders), and chronic infectious processes of the aorta or lung. More recently cases are related to surgical or endovascular procedures of the thoracic aorta [1]. If the presentation of the aorto-pulmonary artery fistula was chronic, we would expect to see signs and symptoms of pulmonary hypertension and congestive heart failure [2]. This would be carefully planned and managed between the cardiothoracic and vascular surgeons as well as interventional radiologists [3]-[7].

The presentation is extremely rare and usually diagnosed on post mortem due to the sudden onset and highly lethal nature of the condition [8].

In the present case we hypothesize following three possible causes. Firstly because of the knots of polypropylene suture at the level of commisures could erode the aorta when AVR is done via continuous sutures (Figure 2). We are using continuous suturing technique with 3 polypropylene sutures (one for each cusp) for past 20 years and no such case has been reported if so. Secondly use of cautery for dissecting plane between aorta and pulmonary artery could have led to thermal injury of the walls leading to weakening, necrosis and fistula formation later on. The third possibility may be an underlying infective pathology over the suture line which is very unlikely in this case as there was no history of fever absence of vegetation and negative culture reports.

There are challenges inherent to the diagnosis and management of this condition even in a tertiary care setting. The symptoms mimic that of stuck aortic valve. The onset is almost entirely sudden and abrupt and a high index of suspicion is required for its prompt diagnosis by CT angiography and echocardiography [9]. Our case received a chance at diagnoses and management because of patient being in a tertiary care set up at the time of onset of symptoms. If this life threatening condition occurs outside of the hospital settings chances of survival remain meager.

There are several limitations to our analysis of the case report. Firstly the relatives of the patient did not consent to an autopsy. Identification of vegetations in the pulmonary or systemic circulation would have supported our hypothesis for an infective pathology although post operative blood cultures also showed no growth. In management, to prolong survival patient could have been supported with ECMO however the facility was not available in our settings.

\section{Conclusion}

We report an exceedingly rare phenomenon of an aorto-pulmonary artery fistula in a follow-up case of post aortic valve replacement. We postulated this was due to infective or iatrogenic cause which involved the aorta and

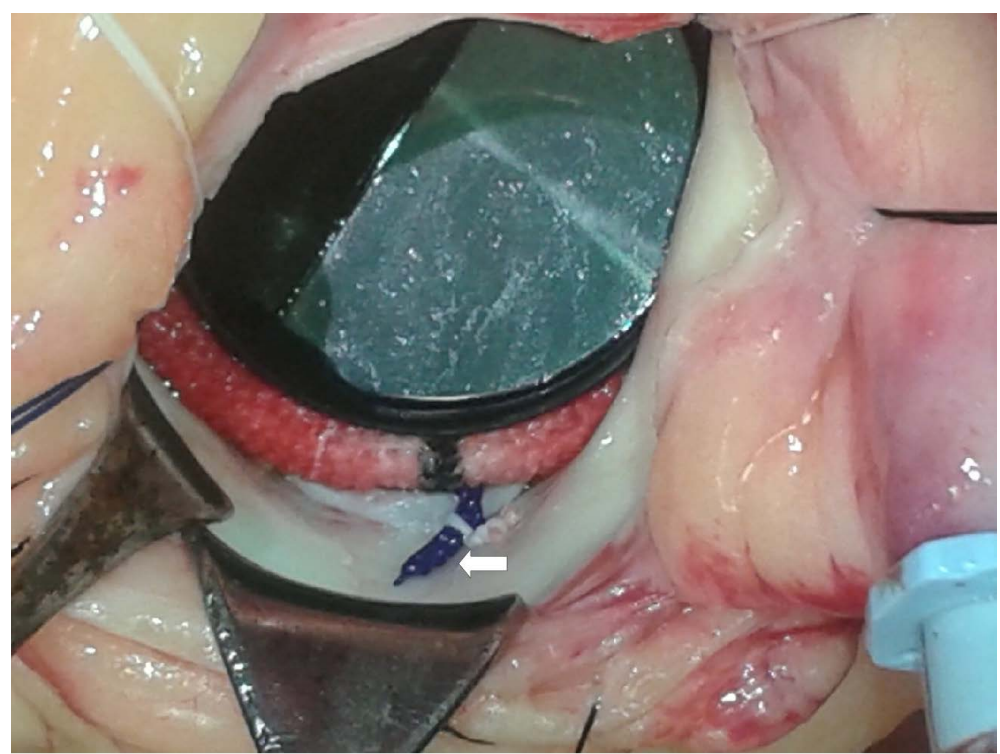

Figure 2. Knot of polypropylene suture (arrow) which may have resulted in aortic erosion and fistula formation (picture taken from different case). 
the main pulmonary artery. This case highlights the difficulty in prompt and accurate diagnosis preoperatively and acute management of the condition. Also we could not establish the cause of its occurrence. Further case series on this condition are essential to understand management strategies and chances of survival as well as to hypothesize the etiology.

\section{References}

[1] Mukadam, M., Barraclough, J., Riley, P. and Bonser, R. (2005) Acquired Aorto-Pulmonary Artery Fistula Following Proximal Aortic Surgery. Interactive Cardiovascular and Thoracic Surgery, 4, 388-390. http://dx.doi.org/10.1510/icvts.2005.106245

[2] Nicholson, R.E. (1943) Syndrome of Rupture of Aortic Aneurysm into Thepulmonary Artery: Review of the Literature with Report of Two Cases. Annals of Internal Medicine, 19, 286-325. http://dx.doi.org/10.7326/0003-4819-19-2-286

[3] Massetti, M., Babatasi, G., Rossi, A., Kapadia, N., Neri, E., Bhoyroo, S., et al. (1995) Aorto-Pulmonary Fistula: An Uncommon Complication in Dystrophic Aortic Aneurysm. The Annals of Thoracic Surgery, 59, 1563-1564. http://dx.doi.org/10.1016/0003-4975(95)00032-G

[4] Lahey, S.J. (1993) Successful Surgical Management of an Aortic Arch Aneurysm with Acute Aorto-Pulmonary Fistula. The Annals of Thoracic Surgery, 55, 995-997. http://dx.doi.org/10.1016/0003-4975(93)90134-4

[5] Coselli, J.S., Le Maire, S.A. and van Cleve, G.D. (1995) Rupture of a Dissecting Thoracic Aortic Aneurysm into the Pulmonary Artery: Successful Surgical Repair. Cardiovascular Surgery, 3, 697-701. http://dx.doi.org/10.1016/0967-2109(96)82872-9

[6] Kochi, K., Okada, K., Watari, M., Orihashi, K. and Sueda, T. (2002) Hybrid Endovascular Stent Grafting for Aortic Arch Aneurysm with Aorto-Pulmomary Fistula. Journal of Thoracic and Cardiovascular Surgery, 123, 363-364. http://dx.doi.org/10.1067/mtc.2002.119061

[7] Ailawadi, G., Lim, D.S., Peeler, B.B., Matsumoto, A.H. and Dake, M.D. (2007) Traumatic Ascending Aortopulmonary Window Following Pulmonary Artery Stent Dilatation: Therapy with Aortic Endovascular Stent Graft. Pediatric Cardiology, 28, 305-308. http://dx.doi.org/10.1007/s00246-006-0118-9

[8] Nghiem, A.Z., Wormald, J.R. and Villar, S.R. (2014) An Interesting Case of an Aortopulmonary Artery Fistula. Journal of Surgical Case Reports, 2014, Article ID: rju079.

[9] Lempel, J.K., Bauman, J.S. and White, C.S. (2012) Aorto-Pulmonary Fistula in Acute Dissection: Findings at Unenhanced and Enhanced Computed Tomographic Imaging. Journal of Thoracic Imaging, 27, 168-170. http://dx.doi.org/10.1097/RTI.0b013e31822d9a86 